\title{
REVIEW
}

\section{New and conventional strategies for lung recruitment in acute respiratory distress syndrome}

\author{
Paolo Pelosi*1 ${ }^{* 1}$ Marcelo Gama de Abreu² and Patricia RM Rocco ${ }^{3}$ \\ This article is one of ten reviews selected from the Yearbook of Intensive Care and Emergency Medicine 2010 (Springer Verlag) and co-published \\ as a series in Critical Care. Other articles in the series can be found online at http://ccforum/series/yearbook. Further information about the \\ Yearbook of Intensive Care and Emergency Medicine is available from http://www.springer.com/series/2855.
}

\begin{abstract}
Introduction
Mechanical ventilation is a supportive and life saving therapy in patients with acute lung injury (ALI)/acute respiratory distress syndrome (ARDS). Despite advances in critical care, mortality remains high [1]. During the last decade, the fact that mechanical ventilation can produce morphologic and physiologic alterations in the lungs has been recognized [2]. In this context, the use of low tidal volumes $\left(\mathrm{V}_{\mathrm{T}}\right)$ and limited inspiratory plateau pressure (Pplat) has been proposed when mechanically ventilating the lungs of patients with ALI/ARDS, to prevent lung as well as distal organ injury [3]. However, the reduction in $\mathrm{V}_{\mathrm{T}}$ may result in alveolar derecruitment, cyclic opening and closing of atelectatic alveoli and distal small airways leading to ventilator-induced lung injury (VILI) if inadequate low positive end-expiratory pressure (PEEP) is applied [4]. On the other hand, high PEEP levels may be associated with excessive lung parenchyma stress and strain [5] and negative hemodynamic effects, resulting in systemic organ injury [6]. Therefore, lung recruitment maneuvers have been proposed and used to open up collapsed lung, while PEEP counteracts alveolar derecruitment due to low $V_{T}$ ventilation [4]. Lung recruitment and stabilization through use of PEEP are illustrated in Figure 1. Nevertheless, the beneficial effects of recruitment maneuvers in ALI/ARDS have been questioned. Although Hodgson et al. [7] showed no evidence that recruitment maneuvers reduce mortality or the duration of mechanical ventilation in patients with ALI/ARDS, such maneuvers may be useful to reverse lifethreatening hypoxemia [8] and to avoid derecruitment
\end{abstract}

${ }^{*}$ Correspondence: ppelosi@hotmail.com

'Department of Ambient Health and Safety, Servizio Anestesia B, Ospedale di Circolo, University of Insubria, Viale Borri 57, 21100 Varese, Italy

Full list of author information is available at the end of the article resulting from disconnection and/or airway suctioning procedures [9].

The success and/or failure of recruitment maneuvers are associated with various factors: 1) Different types of lung injury, mainly pulmonary and extra-pulmonary origin; 2) differences in the severity of lung injury; 3 ) the transpulmonary pressures reached during recruitment maneuvers; 4) the type of recruitment maneuver applied; 5) the PEEP levels used to stabilize the lungs after the recruitment maneuver; 6) differences in patient positioning (most notably supine vs prone); 7) use of different vasoactive drugs, which may affect cardiac output and the distribution of pulmonary blood flow, thus modifying gas-exchange.

Although numerous reviews have addressed the use of recruitment maneuvers to optimize ventilator settings in ALI/ARDS, this issue remains controversial. While some types of recruitment maneuver have been abandoned in clinical practice, new, potentially interesting strategies able to recruit the lungs have not been properly considered. In the present chapter we will describe and discuss: a) Definition and factors affecting recruitment; b) types of recruitment maneuvers; and c) the role of variable ventilation as a recruitment maneuver.

\section{Definition and factors affecting recruitment maneuvers}

Recruitment maneuver denotes the dynamic process of an intentional transient increase in transpulmonary pressure aimed at opening unstable airless alveoli, which has also been termed alveolar recruitment maneuver. Although the existence of alveolar closure and opening in ALI/ARDS has been questioned [10], the rationale for recruitment maneuvers is to open the atelectatic alveoli, thus increasing endexpiratory lung volume, improving gas exchange, and attenuating VILI [11]. However, 


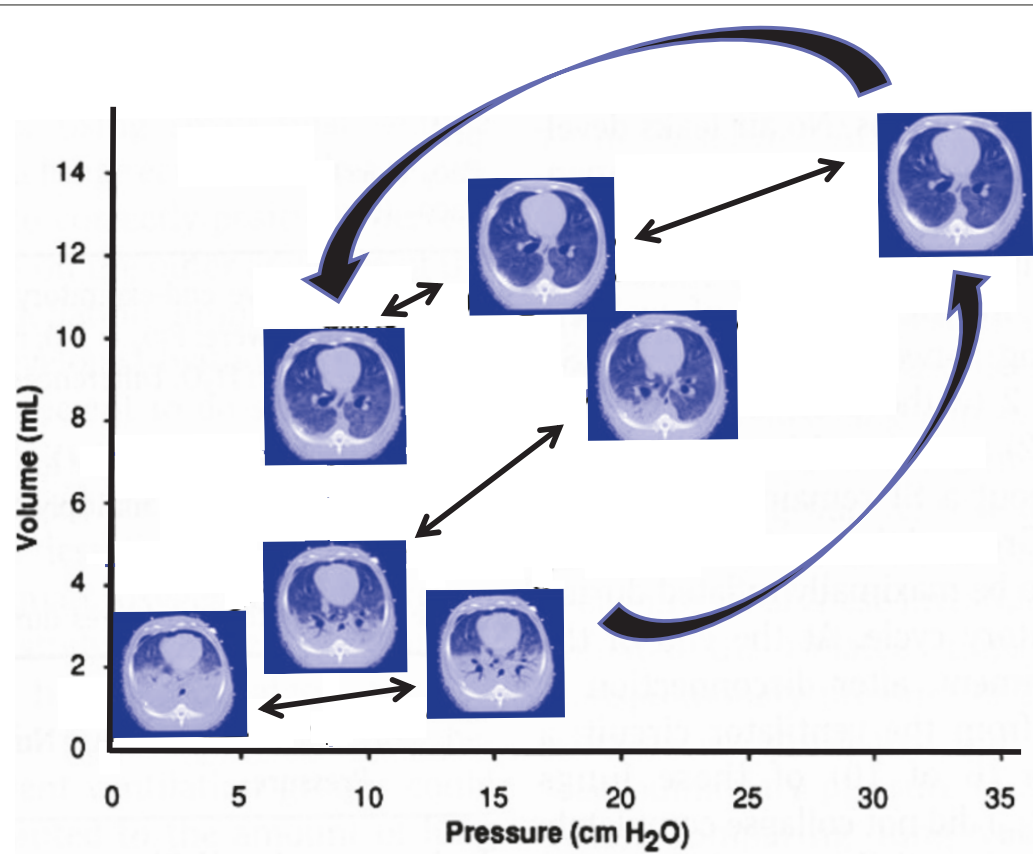

Figure 1. Computed tomography images of oleic acid-induced acute lung injury in dogs at different inspiratory and expiratory pressures. Note the improvement in alveolar aeration at end-expiration after the recruitment maneuver. Large arrows represent inspiration and expiration. Double-ended arrows represent the tidal breathing (end-expiration and end-inspiration). Adapted from [4].

recruitment maneuvers may also contribute to VILI [11, 12], with translocation of pulmonary bacteria [13] and cytokines into the systemic circulation [14]. Furthermore, since recruitment maneuvers increase mean thoracic pressure, they may lead to a reduction in venous return with impairment of cardiac output [15].

Various factors may influence the response to a recruitment maneuver, namely: 1 ) The nature and extent of lung injury, and 2) patient positioning.

\section{Nature and extent of lung injury}

The nature of the underlying injury can affect the response to a recruitment maneuver. In direct (pulmonary) lung injury, the primary structure damaged is the alveolar epithelium resulting in alveolar filling by edema, fibrin, and neutrophilic aggregates. In indirect (extrapulmonary) lung injury, inflammatory mediators are released from extrapulmonary foci into the systemic circulation leading to microvessel congestion and interstitial edema with relative sparing of intra-alveolar spaces [16]. Therefore, recruitment maneuvers should be more effective to open atelectatic lung regions in indirect compared to direct lung injury. Based on this hypothesis, Kloot et al. [17] investigated the effects of recruitment maneuvers on gas exchange and lung volumes in three experimental models of ALI: Saline lavage or surfactant depletion, oleic acid, and pneumonia, and observed improvement in oxygenation only in ALI induced by surfactant depletion. Riva et al. [18] compared the effects of a recruitment maneuver in models of pulmonary and extrapulmonary ALI, induced by intratracheal and intraperitoneal instillation of Escherichia coli lipopolysaccharide, with similar transpulmonary pressures. They found that the recruitment maneuver was more effective for opening collapsed alveoli in extrapulmonary compared to pulmonary ALI, improving lung mechanics and oxygenation with limited damage to alveolar epithelium. Using electrical impedance and computed tomography (CT) to assess lung ventilation and aeration, respectively, Wrigge et al. [19] suggested that the distribution of regional ventilation was more heterogeneous in extrapulmonary than in pulmonary ALI during lung recruitment with slow inspiratory flow. However, this phenomenon and the claim that recruitment maneuvers are useful to protect the so called 'baby lung', i.e., the lung tissue that is usually present in ventral areas and receives most of the tidal ventilation, has been recently challenged. According to Grasso et al. [20], recruitment maneuvers combined with high PEEP levels can lead to hyperinflation of the baby lung due to inhomogeneities in the lung parenchyma, independent of the origin of the injury (pulmonary or extrapulmonary).

Recently, we assessed the impact of recruitment maneuvers on lung mechanics, histology, inflammation and fibrogenesis at two different degrees of lung injury (moderate and severe) in a paraquat ALI model [21]. 
While both degrees of injury showed comparable amounts of lung collapse, severe ALI was accompanied by alveolar edema. After a recruitment maneuver, lung mechanics improved and the amount of atelectasis was reduced to similar extents in both groups, but in the presence of alveolar edema, the recruitment maneuver led to hyperinflation, and triggered an inflammatory as well as a fibrogenic response in the lung tissue.

\section{Patient positioning}

Prone positioning may not only contribute to the success of recruitment maneuvers, but should itself be considered as a recruitment maneuver. In the prone position, the transpulmonary pressure in dorsal lung areas increases, opening alveoli and improving gasexchange [22]. Some authors have reported that in healthy [23], as well as in lung-injured animals [24], mechanical ventilation leading to lung overdistension and cyclic collapse/reopening was associated with less extensive histological change in dorsal regions in the prone, as compared to the supine position. Although the claim that body position affects the distribution of lung injury has been challenged, the development of VILI due to excessively high $\mathrm{V}_{\mathrm{T}}$ seems to be delayed during prone compared to supine positioning [25].

The reduction or delay in the development of VILI in the prone position can be explained by different mechanisms: (a) A more homogeneous distribution of transpulmonary pressure gradient due to changes in the lung-thorax interactions and direct transmission of the weight of the abdominal contents and heart [22], yielding a redistribution of ventilation; (b) increased endexpiratory lung volume resulting in a reduction in stress and strain [25]; and (c) changes in regional perfusion and/or blood volume [26]. In a paraquat model of ALI, the prone position was associated with a better perfusion in ventral and dorsal regions, a more homogeneous distribution of alveolar aeration which reduced lung mechanical changes and increased end expiratory lung volume and oxygenation [27]. In addition, the prone position reduced alveolar stress but no regional changes were observed in inflammatory markers. Recruitment maneuvers also improved oxygenation more effectively with a decreased PEEP requirement for preservation of the oxygenation response in prone compared with supine position in oleic acid-induced lung injury [28]. Those findings suggest that the prone position may protect the lungs against VILI, and recruitment maneuvers can be more effective in the prone compared to the supine position.

\section{Types of recruitment maneuver}

A wide variety of recruitment maneuvers has been described. The most relevant are represented by: Sustained inflation maneuvers, high pressure controlled ventilation, incremental PEEP, and intermittent sighs. However, the best recruitment maneuver technique is currently unknown and may vary according to the specific circumstances.

The most commonly used recruitment maneuver is the sustained inflation technique, in which a continuous pressure of $40 \mathrm{cmH}_{2} \mathrm{O}$ is applied to the airways for up to $60 \mathrm{sec}$ [8]. Sustained inflation has been shown to be effective in reducing lung atelectasis [29], improving oxygenation and respiratory mechanics $[18,29]$, and preventing endotracheal suctioning-induced alveolar derecruitment [9]. However, the efficacy of sustained inflation has been questioned and other studies showed that this intervention may be ineffective [30], short-lived [31], or associated with circulatory impairment [32], an increased risk of baro/volutrauma [33], a reduced net alveolar fluid clearance [34], or even worsened oxygenation [35].

In order to avoid such side effects, other types of recruitment maneuver have been developed and evaluated. The most important are: 1) incrementally increased PEEP limiting the maximum inspiratory pressure [36]; 2) pressure-controlled ventilation applied with escalating PEEP and constant driving pressure [30]; 3) prolonged lower pressure recruitment maneuver with PEEP elevation up to $15 \mathrm{cmH}_{2} \mathrm{O}$ and end inspiratory pauses for $7 \mathrm{sec}$ twice per minute during 15 min [37]; 4) intermittent sighs to reach a specific plateau pressure in volume or pressure control mode [38]; and 5) long slow increase in inspiratory pressure up to $40 \mathrm{cmH}_{2} \mathrm{O}$ (RAMP) [18].

\section{Impact of recruitment maneuver on ventilator- induced lung injury}

While much is known about the impact of recruitment maneuvers on lung mechanics and gas exchange, only a few studies have addressed their effects on VILI. Recently, Steimback et al. [38] evaluated the effects of frequency and inspiratory plateau pressure (Pplat) during recruitment maneuvers on lung and distal organs in rats with ALI induced by paraquat. They observed that although a recruitment maneuver with standard sigh (180 sighs/ hour and Pplat $=40 \mathrm{cmH}_{2} \mathrm{O}$ ) improved oxygenation and decreased $\mathrm{PaCO}_{2}$, lung elastance, and alveolar collapse, it resulted in hyperinflation, ultrastructural changes in alveolar capillary membrane, increased lung and kidney epithelial cell apoptosis, and type III procollagen (PCIII) mRNA expression in lung tissue. On the other hand, reduction in the sigh frequency to 10 sighs/hour at the same Pplat $\left(40 \mathrm{cmH}_{2} \mathrm{O}\right)$ diminished lung elastance and improved oxygenation, with a marked decrease in alveolar hyperinflation, PCIII mRNA expression in lung tissue, and apoptosis in lung and kidney epithelial cells. 


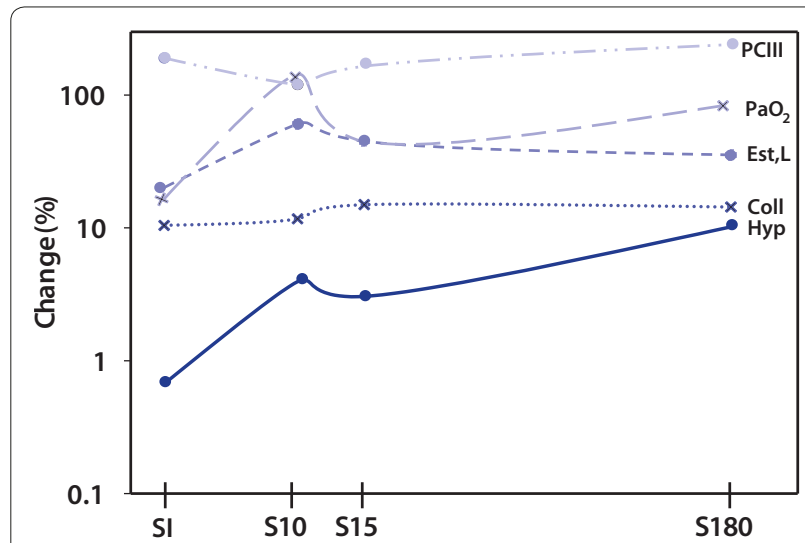

Figure 2. Percentage of change in static lung elastance (Est,L), oxygenation $\left(\mathrm{PaO}_{2}\right)$, fractional area of alveolar collapse (Coll) and hyperinflation (Hyp), and mRNA expression of type III procollagen (PCIII) from sustained inflation (SI) and sigh at different frequencies (10, 15 and 180 per hour) to non-recruited acute lung injury rats. Note that at low sigh frequency, oxygenation and lung elastance improved, followed by a reduction in alveolar collapse and PCIII. Adapted from [38].

However, the association of this sigh frequency with a lower Pplat of $20 \mathrm{cmH}_{2} \mathrm{O}$ worsened lung elastance, histology and oxygenation, and increased $\mathrm{PaCO}_{2}$ with no modifications in PCIII mRNA expression in lung tissue and epithelial cells apoptosis of distal organs. Figure 2 illustrates some of these effects. We speculate that there is a sigh frequency threshold beyond which the intrinsic reparative properties of the lung epithelium are overwhelmed. Although the optimal sigh frequency may be different in healthy animals/patients compared to those with ALI, our results suggest that recruitment maneuvers with high frequency or low plateau pressure should be avoided. Theoretically, a recruitment maneuver using gradual inflation of the lungs may yield a more homogeneous distribution of pressure throughout the lung parenchyma, avoiding repeated maneuvers and reducing lung stretch while allowing effective gas exchange.

Riva et al. [18] compared the effects of sustained inflation using a rapid high recruitment pressure of $40 \mathrm{cmH}_{2} \mathrm{O}$ for $40 \mathrm{sec}$ with a progressive increase in airway pressure up to $40 \mathrm{cmH}_{2} \mathrm{O}$ reached at $40 \mathrm{sec}$ after the onset of inflation (so called RAMP) in paraquat-induced ALI. They reported that the RAMP maneuver improved lung mechanics with less alveolar stress. Among other recruitment maneuvers proposed as alternatives to sustained inflation, RAMP may differ according to the time of application and the mean airway pressure.

Recently, Saddy and colleagues [39] reported that assisted ventilation modes such as assist-pressure controlled ventilation (APCV) and biphasic positive airway pressure associated with pressure support Ventilation (BiVent+PSV) led to alveolar recruitment improving gas-exchange and reducing inflammatory and fibrogenic mediators in lung tissue compared to pressure controlled Ventilation. They also showed that BiVent+PSV was associated with less inspiratory effort, reduced alveolar capillary membrane injury, and fewer inflammatory and fibrogenic mediators compared to APCV [39].

\section{The role of variable ventilation as a recruitment maneuver}

Variable mechanical ventilation patterns are characterized by breath-by-breath changes in $\mathrm{V}_{\mathrm{T}}$ that mimic spontaneous breathing in normal subjects, and are usually accompanied by reciprocal changes in the respiratory rate. Time series of $\mathrm{V}_{\mathrm{T}}$ and respiratory rate values during variable mechanical ventilation may show longrange correlations, which are more strictly 'biological', or simply random (noisy). Both biological and noisy patterns of variable mechanical ventilation have been shown to improve oxygenation and respiratory mechanics, and reduce diffuse alveolar damage in experimental ALI/ ARDS [40, 41]. Although different mechanisms have been postulated to explain such findings, lung recruitment seems to play a pivotal role.

Suki et al. [42] showed that once the critical opening pressure of collapsed airways/alveoli was exceeded, all subtended or daughter airways/alveoli with lower critical opening pressure would be opened in an avalanche. Since the critical opening pressure values of closed airways as well as the time to achieve those values may differ through the lungs, mechanical ventilation patterns that produce different airway pressures and inspiratory times may be advantageous to maximize lung recruitment and stabilization, as compared to regular patterns. Accordingly, variable controlled mechanical ventilation has been reported to improve lung function in experimental models of atelectasis [43] and during one-lung ventilation [44]. In addition, Boker et al. [45] reported improved arterial oxygenation and compliance of the respiratory system in patients ventilated with variable compared to conventional mechanical ventilation during surgery for repair of abdominal aortic aneurysms, where atelectasis is likely to occur due to increased intra-abdominal pressure.

There is increasing experimental evidence suggesting that variable mechanical ventilation represents a more effective way of recruiting the lungs than conventional recruitment maneuvers. Bellardine et al. [46] showed that recruitment following high $V_{T}$ ventilation lasted longer with variable than with monotonic ventilation in excised calf lungs. In addition, Thammanomai et al. [47] showed that variable ventilation improved recruitment in normal and injured lungs in mice. In an experimental lavage model of ALI/ARDS, we recently showed that oxygenation improvement following a recruitment 


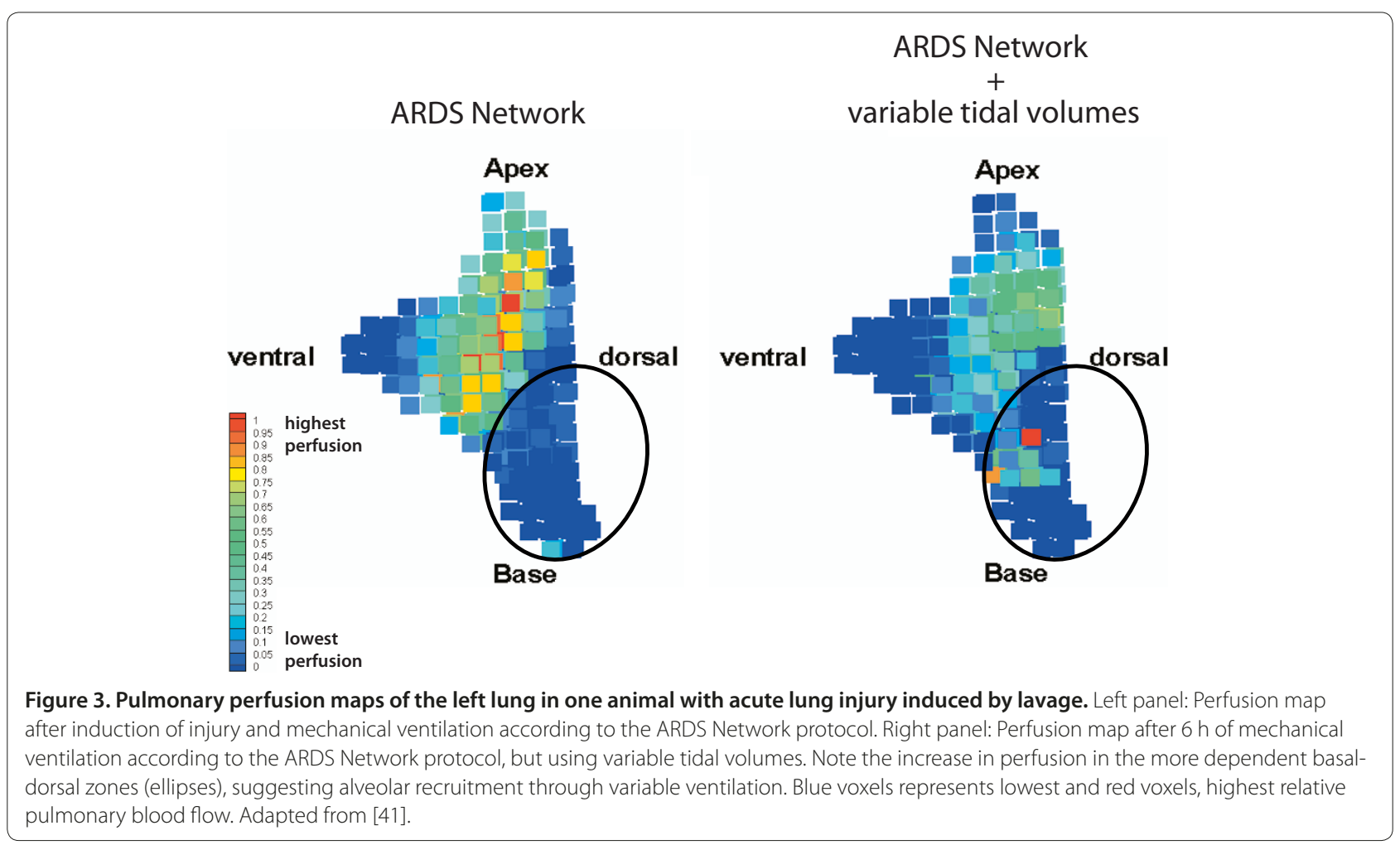

maneuver through sustained inflation was more pronounced when combined with variable mechanical ventilation [41]. Additionally, the redistribution of pulmonary blood flow from cranial to caudal and from ventral to dorsal lung zones was higher and diffuse alveolar damage less when variable ventilation was associated with the ventilation strategy recommended by the ARDS Network. Such a redistribution pattern of pulmonary perfusion, which is illustrated in Figure 3, is compatible with lung recruitment [41].

The phenomenon of stochastic resonance may explain the higher efficiency of variable ventilation as a recruitment maneuver. In non-linear systems, like the respiratory system, the amplitude of the output can be modulated by the noise in the input. Typical inputs are driving pressure, $\mathrm{V}_{\mathrm{T}}$, and respiratory rate, while outputs are the mechanical properties, lung volume, and gas exchange. Thus, by choosing appropriate levels of variability (noise) in $\mathrm{V}_{\mathrm{T}}$ during variable volume controlled ventilation, or in driving pressure during variable pressure controlled ventilation [48], the recruitment effect can be optimized.

Despite the considerable amount of evidence regarding the potential of variable ventilation to promote lung recruitment, this mechanism is probably less during assisted ventilation. In experimental ALI, we showed that noisy pressure support ventilation (noisy PSV) improved oxygenation $[49,50]$, but this effect was mainly related to lower mean airway pressures and redistribution of pulmonary blood flow towards better ventilated lung zones.

\section{Conclusion}

In patients with ALI/ARDS, considerable uncertainty remains regarding the appropriateness of recruitment maneuvers. The success/failure of such maneuvers may be related to the nature, phase, and/or extent of the lung injury, as well as to the specific recruitment technique. At present, the most commonly used recruitment maneuver is the conventional sustained inflation, which may be associated with marked respiratory and cardiovascular adverse effects. In order to minimize such adverse effects, a number of new recruitment maneuvers have been suggested to achieve lung volume expansion by taking into account the level and duration of the recruiting pressure and the pattern/frequency with which this pressure is applied to accomplish recruitment. Among the new types of recruitment maneuver, the following seem particularly interesting: 1) incremental increase in PEEP limiting the maximum inspiratory pressure; 2) pressure-controlled ventilation applied with escalating PEEP and constant driving pressure; 3) prolonged lower pressure recruitment maneuver with PEEP elevation up to $15 \mathrm{cmH}_{2} \mathrm{O}$ and end-inspiratory pauses for $7 \mathrm{sec}$ twice per minute during $15 \mathrm{~min}$; 4) intermittent sighs to reach a specific plateau pressure in volume or pressure control mode; and 5) long slow increase in inspiratory pressure 
up to $40 \mathrm{cmH}_{2} \mathrm{O}$ (RAMP). Moreover, the use of variable controlled ventilation, i.e., application of breath-by-breath variable $\mathrm{V}_{\mathrm{T}} \mathrm{s}$ or driving pressures, as well as assisted ventilation modes such as Bi-Vent+PSV, may also prove a simple and interesting alternative for lung recruitment in the clinical scenario. Certainly, comparisons of different lung recruitment strategies and randomized studies to evaluate their impact on morbidity and mortality are warranted in patients with ALI/ARDS.

\section{Abbreviations}

$\mathrm{ALI}=$ acute lung injury, APCV = assist-pressure controlled ventilation, ARDS = acute respiratory distress syndrome, $\mathrm{CT}=$ computed tomography, PSV = pressure support ventilation, $\mathrm{PEEP}=$ positive end-expiratory pressure, $\mathrm{PCIII}=$ type III procollagen, Pplat = plateau pressure, VILI = ventilator-induced lung injury, $V_{T}=$ tidal volume.

\section{Author details}

'Department of Ambient Health and Safety, Servizio Anestesia B, Ospedale di Circolo, University of Insubria, Viale Borri 57, 21100 Varese, Italy

${ }^{2}$ Department of Anesthesiology and Intensive Care, Pulmonary Engineering Group, University Hospital Carl Gustav Carus, Fetscherstr. 74, 01307 Dresden, Germany

${ }^{3}$ Laboratory of Pulmonary Investigation, Universidade Federal do Rio de Janeiro, Instituto de Biofisica Carlos Chagas Filho, C.C.S. Ilha do Fundao, $21941-$ 902 Rio de Janeiro, Brazil

\section{Competing interests}

MGdA - Drager Medical AG (Lübeck Germany) provided MGdA with the mechanical ventilator and technical assistance to perform the variable pressure support ventilation mode that is mentioned in this manuscript. MGdA has been granted patents on the variable pressure support mode of assisted ventilation and on a controller for adjusting variable pressure support ventilation in presence of intrinsic variability of the breath pattern. PP and PRMR declare that they have no competing interests.

Published: 9 March 2010

\section{References}

1. Phua J, Badia JR, Adhikari NKJ, et al:: Has mortality from acute respiratory distress syndrome decreased over time? Am J Respir Crit Care Med 2009, 179:220-227.

2. Oeckler RA, Hubmayr RD: Ventilator-associated lung injury: a search for better therapeutic targets. Eur Respir J 2007, 30:1216-1226.

3. The Acute Respiratory Distress Syndrome Network: Ventilation with lower tidal volumes as compared with traditional tidal volumes for acute lung injury and the acute respiratory distress syndrome. N Engl J Med 2000, 342(18):1301-1308.

4. Pelosi P, Goldner M, McKibben A, et al:: Recruitment and derecruitment during acute respiratory failure: an experimental study. Am J Respir Crit Care Med 2001, 164:122-130.

5. Pássaro CP, Silva PL, Rzezinski AF, et al.: Pulmonary lesion induced by low and high positive end-expiratory pressure levels during protective ventilation in experimental acute lung injury. Crit Care Med 2009,37:1011-1017.

6. Imai Y, Parodo J, Kajikawa O, et al.: Injurious mechanical ventilation and end-organ epithelial cell apoptosis and organ dysfunction in an experimental model of acute respiratory distress syndrome. JAMA 2003, 289:2104-2112.

7. Hodgson C, Keating JL, Holland AE, et al.: Recruitment manoeuvres for adults with acute lung injury receiving mechanical ventilation. Cochrane Database Syst Rev 2009,15:CD006667.

8. Fan E, Wilcox ME, Brower RG, et al.: Recruitment maneuvers for acute lung injury: a systematic review. Am J Respir Crit Care Med 2008,178:1156-1163.

9. Maggiore SM, Lellouche F, Pigeot J, et al:: Prevention of endotracheal suctioning-induced alveolar rerecruitment in acute lung injury. Am J Respir Crit Care Med 2003, 167:1215-1224.

10. Martynowicz MA, Walters BJ, Hubmayr RD: Mechanisms of recruitment in oleic acidinjured lungs. J Appl Physiol 2000, 90:1744-1753.
11. Tremblay LN, Slutsky AS: Ventilator-induced lung injury: from the bench to the bedside. Intensive Care Med 2006, 32:24-33.

12. Gattinoni L, Pesenti A: The concept of "baby lung". Intensive Care Med 2005, 31:776-784.

13. Cakar N, Akinci O, Tugrul S, et al.: Recruitment maneuver: does it promote bacterial translocation? Crit Care Med 2002 30:2103-2106.

14. Halbertsma FJ, Vaneker $M$, Pickkers $P$, et al.: A single recruitment maneuver in ventilated critically ill children can translocate pulmonary cytokines into the circulation. J Crit Care 2009 (in press).

15. Lim SC, Adams AB, Simonson DA, et al:: Transient hemodynamic effects of recruitment maneuvers in three experimental models of acute lung injury. Crit Care Med 2004, 32:2378-2384.

16. Rocco PRM, Pelosi P: Pulmonary and extrapulmonary acute respiratory distress syndrome: myth or reality? Curr Opin Crit Care Med 2008, 14:50-55.

17. Kloot TE, Blanch L, Youngblood MA, et al:: Recruitment maneuvers in three experimental models of acute lung injury. Effect on lung volume and gas exchange. Am J Respir Crit Care Med 2000, 161:1485-1494.

18. Riva DR, Oliveira MB, Rzezinski AF, et al:: Recruitment maneuver in pulmonary and extrapulmonary experimental acute lung injury. Crit Care Med 2009, 36:1900-1908.

19. Wrigge $H$, Zinserling J, Muders T, et al:. Electrical impedance tomography compared with thoracic computed tomography during a slow inflation maneuver in experimental models of lung injury. Crit Care Med 2008 36:903-909.

20. Grasso S, Stripoli, T, Sacchi M, et al:: Inhomogeneity of lung parenchyma during the open lung strategy: a computed tomography scan study. Am J Respir Crit Care Med 2009,180:415-423.

21. Ornellas D, Santiago VR, Rzezinski AF, et al.: Lung mechanical stress induced by recruitment maneuver in different degrees of acute lung injury [abstract]. Am J Respir Crit Care Med 2009,179:A3837.

22. Mutoh T, Guest RJ, Lamm WJE, Albert RK: Prone position alters the effect of volume overload on regional pleural pressures and improves hypoxemia in pigs invivo. Am Rev Respir Dis 1992,146:300-306.

23. Nakos G, Batistatou A, Galiatsou E, et al.: Lung and 'end organ' injury due to mechanical ventilation in animals: comparison between the prone and supine positions. Crit Care 2006, 10:R38.

24. Broccard AF, Shapiro RS, Schmitz LL, Ravenscraft SA, Marini JJ: Influence of prone position on the extent and distribution of lung injury in a high tidal volume oleic acid model of acute respiratory distress syndrome. Crit Care Med 1997, 25:16-27.

25. Valenza F, Guglielmi M, Maffioletti M, et al.: Prone position delays the progression of ventilator-induced lung injury in rats: does lung strain distribution play a role? Crit Care Med 2005 33:361-367.

26. Richter T, Bellami G, Scott Harris R, et al.: Effect of prone position on regional shunt, aeration, and perfusion in experimental acute lung injury. Am J Respir Crit Care Med 2005, 172:480-487.

27. Santana MC, Garcia CS, Xisto DG, et al:: Prone position prevents regional alveolar hyperinflation and mechanical stress and strain in mild experimental acute lung injury. Respir Physio/ Neurobio/ 2009,167:181-188.

28. Cakar N, der Kloot TV, Youngblood M, Adams A, Nahum A: Oxygenation response to a recruitment maneuver during supine and prone positions in an oleic acid-induced lung injury model. Am J Respir Crit Care Med 2000 161:1949-1956.

29. Farias LL, Faffe DS, Xisto DG, et al:: Positive end-expiratory pressure prevents lung mechanical stress caused by recruitment/derecruitment. J App/ Physiol, 2005 98:53-61.

30. Villagrá A, Ochagavía A, Vatua S, et al.: Recruitment maneuvers during lung protective ventilation in acute respiratory distress syndrome. Am J Respir Crit Care Med 2002 165:165-170.

31. Brower RG, Morris A, Maclntyre N, et al:: Effects of recruitment maneuvers in patients with acute lung injury and acute respiratory distress syndrome ventilated with high positive end-expiratory pressure. Crit Care Med 2003 31:2592-2597.

32. Odenstedt $\mathrm{H}$, Aneman A, Kárason S, Stenqvist O, Lundin S: Acute hemodynamic changes during lung recruitment in lavage and endotoxininduced ALI. Intensive Care Med 2005, 31:112-120.

33. Meade MO, Cook DJ, Griffith LE, et al:: A study of the physiologic responses to a lung recruitment maneuver in acute lung injury and acute respiratory distress syndrome. Respir Care 2008 53:1441-1449.

34. Constantin JM, Cayot-Constantin S, Roszyk L, et al:: Response to recruitment maneuver influenc.es net al.:veolar fluid clearance in acute respiratory 
distress syndrome. Anesthesiology 2007 106:944-951.

35. Musch G, Harris RS, Vidal Melo MF, et al:: Mechanism by which a sustained inflation can worsen oxygenation in acute lung injury. Anesthesiology 2004, 100:323-330.

36. Rzezinski AF, Oliveira GP, Santiago VR, et al:: Prolonged recruitment manoeuvre improves lung function with less utrastructural damage in experimental mild acute lung injury. Respir Physio/ Neurobiol 2009 169:271-281.

37. Odenstedt $H$, Lindgren $\mathrm{S}$, Olegard $\mathrm{C}$, et al.: Slow moderate pressure recruitment maneuver minimizes negative circulatory and lung mechanic side effects: evaluation of recruitment maneuvers using electric impedance tomography. Intensive Care Med 2005, 31:1706-1714.

38. Steimback PW, Oliveira GP, Rzezinksi AF, et al.: Effects of frequency and inspiratory plateau pressure during recruitment manoeuvres on lung and distal organs in acute lung injury. Intensive Care Med 2009, 35:1120-1128.

39. Saddy F, Oliveira GP, Garcia CS, et al:: Assisted ventilation modes reduce the expression of lung inflammatory and fibrogenic mediators in a model of mild acute lung injury. Intensive Care Med 2010, (in press).

40. Funk DJ, Graham MR, Girling LG, et al:: A comparison of biologically variable ventilation to recruitment manoeuvres in a porcine model of acute lung injury. Respir Res 2004, 5:22.

41. Spieth PM, Carvalho AR, Pelosi P, et al.: Variable tidal volumes improve lung protective ventilation strategies in experimental lung injury. Am J Respir Crit Care Med 2009, 179:684-693.

42. Suki B, Barabási AL, Hantos Z, Peták F, Stanley HE: Avalanches and power-law behaviour in lung inflation. Nature 1994, 368:615-618.

43. Mutch WAC, Harms S, Graham MR, Kowalski SE, Girling LG, Lefevre GR: Biologically variable or naturally noisy mechanical ventilation recruits atelectatic lung. Am J Respir Crit Care Med 2000, 162:319-323.
44. McMullen MC, Girling LG, Graham MR, Mutch WAC: Biologically variable ventilation improves oxygenation and respiratory mechanics during one-lung ventilation. Anesthesiology 2006,105:91-97.

45. Boker A, Haberman CJ, Girling L, et al:: Variable ventilation improves perioperative lung function in patients undergoing abdominal aortic aneurysmectomy. Anesthesiology 2004, 100:608-616.

46. Bellardine $\mathrm{CL}$, Hoffman AM, Tsai L, Ingenito EP, Arold SP, Lutchen KR, Suki B: Comparison of variable and conventional ventilation in a sheep saline lavage lung injury model. Crit Care Med 2006, 34:439-445.

47. Thammanomai A, Hueser E, Majumdar A, Bartolák-Suki E, Suki B: Design of a new variable-ventilation method optimized for lung recruitment in mice J Appl Physio/ 2008, 104:1329-1340.

48. Beda A, Spieth PM, Handzsuj T, et al:: A novel adaptive control system for noisy pressure controlled ventilation: A numerical stimulation and bench test study. Intensive Care Med 2010, (in press).

49. Gama de Abreu M, Spieth P, Pelosi P, et al:: Noisy pressure support ventilation: A pilot study on a new assisted ventilation mode in experimental lung injury. Crit Care Med 2008, 36:818-827.

50. Spieth P, Carvalho AR, Güldner A, Pelosi P, et al.: Effects of different levels of pressure support variability in experimental lung injury. Anesthesiology 2009, 110: 14-215

doi:10.1186/cc885

Cite this article as: Pelosi $P_{\text {, et }}$ al.: New and conventional strategies for lung recruitment in acute respiratory distress syndrome. Critical Care 2010, 14:210. 